\title{
Working memory capacity modulates task performance but has little influence on task choice
}

\author{
Karin M. Butler • Catherine M. Arrington • \\ Christina Weywadt
}

Published online: 8 December 2010

(C) Psychonomic Society, Inc. 2010

\begin{abstract}
Variation in the ability to maintain internal goals while resolving competition from multiple information streams has been related to individual differences in working memory capacity (WMC). In a multitask environment, task choice and task performance are influenced by internal goals, prior behavior within the environment, and the availability of relevant and irrelevant information in the environment. Using the voluntary task-switching procedure, task performance, as measured by switch costs, was related to WMC, but only at short preparation intervals. Task choice processes were only weakly related to WMC. These findings are consistent with models of cognitive control that separate task choice processes from the processes of activating and maintaining task readiness. WMC is related to regulation of specific task parameters but not to choice processes integral to the coordination of multiple sources of information.
\end{abstract}

Keywords Working memory capacity · Task switching . Choice processing

Mechanisms of cognitive control are central to optimal multitasking performance (Brown, Reynolds, \& Braver 2007;

K. M. Butler $(\bowtie)$

MSC 03 2220; Logan Hall, Department of Psychology,

1 University of New Mexico,

Albuquerque, NM 87131-0001, USA

e-mail: kmbutler@unm.edu

C. M. Arrington

Lehigh University,

Bethlehem, PA, USA

C. Weywadt

University of New Mexico,

Albuquerque, NM, USA
Logan, 1985; Monsell, 2003). Cognitive control must be exerted in a multitask environment because the environment affords multiple behavioral paths and does not specify which task should be completed at any given moment. Guiding the completion of one task in the face of competition from other potential tasks requires the selection and activation of the task set, or group of component cognitive operations, necessary to carry out that task (Rogers \& Monsell, 1995). The goal to perform a given task must be maintained and shielded from interference from other goals potentially applicable in the environment (De Jong, 2000). In addition, as the current task changes, no-longer-relevant task sets are deactivated (Mayr \& Keele, 2000). This type of sequential control requires flexible updating and maintenance of the task rules. Different components of cognitive control are referred to as executive control, executive attention, or working memory.

The demands of a multitasking environment - maintaining goals in the face of competition and distraction and updating goals as the environment changes - are functions that have been attributed to working memory. More specifically, measures of working memory capacity (WMC) are thought to tap the same set of functions. For example, WMC has been defined as "the attentional processes that allow for goaldirected behavior by maintaining relevant information in an active, easily accessible state outside of conscious focus, or to retrieve that information from inactive memory, under conditions of interference, distraction, or conflict" (p. 23; Kane, Conway, Hambrick, \& Engle 2007). Individual differences in WMC are correlated with the abilities to resolve interference between memory representations (Rosen \& Engle, 1998); to filter out distracting information (Conway, Cowan, \& Bunting 2001); to act on the current task goal when habitual attentional orienting may lead to inaccurate responding, as in an antisaccade task (Kane, Bleckley, Conway, \& Engle 2001); and to resolve response competi- 
tion and maintain goals, as in a Stroop task (Kane \& Engle, 2003). Thus, when multitasking is required, the degree of success may be determined in part by individual differences in WMC.

The cognitive control mechanisms involved in multitasking have been studied using task-switching methodologies (see Monsell, 2003; Koch, Gade, Schuch, \& Philipp 2010; Vandierendonck, Liefooghe, \& Verbruggen 2010, for reviews). On each trial, the stimulus display affords multiple tasks. Participants perform the tasks in a specified sequence based on either an instructed order (Logan, 2004; Rogers \& Monsell, 1995) or explicit task cues (Meiran, 1996). The critical independent variable is the task transition from trial to trial. Transitions can be repetitions, where the same task is performed on trial $n$ as on trial $n-1$, or switches, where a different task is performed on the consecutive trials. In this paradigm, the ability to engage cognitive control processes to switch to a new task set is measured by slower and more error-prone performance on switch trials than on repetition trials, measures referred to as local switch costs. Switch costs have been shown to increase under a WM load (Baddeley, Chincotta, \& Adlam 2001; Hester \& Garavan, 2005; but see Liefooghe, Barrouillet, Vandierendonck, \& Camos 2008), supporting the argument that at least some component of switch costs reflects cognitive control mechanisms.

Surprisingly, no studies to date have shown a significant relationship between individual differences in WMC and switch cost measures during task switching (Kane et al., 2007; Miyake et al., 2000; Oberauer, Süß, Schulze, Wilhelm, \& Wittmann 2000; Oberauer, Süß, Wilhelm, \& Wittman 2003). Some have argued that the lack of a correlation is the result of a limitation of the task-switching paradigm (e.g., Kane et al., 2007) because switch costs in cued task-switching paradigms may not reflect cognitive control mechanisms (Allport \& Wylie, 2000; Altmann \& Gray, 2008; Gilbert \& Shallice, 2002). For example, several studies have demonstrated that switch costs can be largely attributed to cue-encoding processes that are faster when a cue repeats from the previous trial (Logan \& Bundesen, 2003; Mayr \& Kliegl, 2003; Schneider \& Logan, 2005). In this view, encoding of the task cue, and not reconfiguration of the processes that guide performance of a new task, causes the switch cost. Thus, if the task performance measures (switch costs) from task-switching paradigms do not reflect cognitive control processes, then the lack of a relationship between WMC and these measures is unremarkable and does little to elucidate the role of WMC in multitask behavior.

In the present study, we examined the relationship between individual differences in WMC and multitasking behavior using a variation on standard task-switching paradigms that was developed to better measure the volitional control processes involved in the choice to switch from one task to another (Arrington \& Logan, 2004, 2005). In the voluntary task-switching procedure (VTS), individuals select which task to perform on each trial. VTS mimics real-world multitask environments in that at each choice point, there is not a right or wrong choice predetermined by the immediate experimental context; rather, the appropriate choice is a function of the history of the individual and the current situation. As in other task-switching procedures, the stimulus display affords two or more tasks, but on any given trial the participants must make a choice about which task to perform without online direction from the experimenter. To ensure some degree of multitasking, participants typically are instructed at the beginning of the experiment to perform the tasks equally often and to select a task at random on each trial. Given these instructions, both the probability of performing either of the two tasks and the probability of switching from one task to the other from trial to trial should be .5. Participants generally do well at performing the tasks with equal probability but have difficulty performing the tasks in a completely random sequence. A prominent repetition bias (i.e., switch probabilities less than .5) is observed in many circumstances (see, e.g., Arrington \& Logan, 2004, 2005; Demanet, Verbruggen, Liefooghe, \& Vandierendonck 2010; Liefooghe, Demanet, \& Vandierendonck 2009).

VTS allows for the consideration of both task performance and task choice measures for assessing the control processes engaged during multitask behavior. Task performance measures such as reaction time (RT) and accuracy are commonly taken as indicators of task readiness and used to examine control processes in multitask environments. However, control is engaged not only in the performance of a behavior, but also in the choice of one behavior over another and in the sequencing of multiple behaviors. Task selection in multitask environments may be distinct from task readiness (Allport \& Wylie, 2000), and as such may engage partially nonoverlapping sets of control processes. While Mayr and Bell (2006) reported correlations between task switch probabilities and switch costs, the correlations have not been replicated in other studies (Arrington \& Yates, 2009).

VTS is argued to require more volitional control than standard task-switching paradigms, in part because of the lack of environmental support (Arrington \& Logan, 2005). In addition, in measures of task choice, the switch probability increases when longer preparation intervals between trials are provided (Arrington \& Logan, 2004; Liefooghe et al., 2009), suggesting that the bias may occur to a greater extent when individuals lack the necessary time to engage in the control processes needed to select a new task randomly and reconfigure the cognitive system to execute it. The switch probability is also negatively 
correlated with a measure of executive control, such that increased probability of switching tasks is associated with a better ability to manage conflict (Arrington \& Yates, 2009). Task performance measures (RTs and accuracy) also may be better indexes of endogenous control in VTS than in cued task switching: given that no task cue is present, switch costs may not be attributable to cue-encoding processes (see, e.g., Logan \& Bundesen, 2003). Further, task readiness effects are more robust in VTS than in cued task switching (Liefooghe et al., 2009).

While a great deal of research using standard taskswitching methodologies has examined the factors that underlie task performance, relatively little is understood about how task selection occurs in VTS. Arrington and Logan (2005) proposed that the ability to keep track of the sequence of tasks performed on the last few trials may be critical for task choice because of use of a representativeness heuristic. Building on an earlier model of random sequence generation (Rapoport \& Budescu, 1997), the representativeness heuristic proposes that individuals maintain in WM a sequence of recently performed tasks and select the task to perform on the upcoming trial such that the sequence will best match an internal representation of randomness. Indeed, it has been shown that task selection is influenced by the contents of WM (Demanet et al., 2010; Weaver \& Arrington, 2010). Use of the representativeness heuristic requires WM resources because it requires maintenance of the task sequence, comparison of that sequence with sequences representative of randomness, and updating of the sequence when a new task is executed. Under conditions in which this more-demanding comparison process cannot be carried out, individuals may rely on an availability heuristic to guide task choice. In this case, the task most available in WM will be selected. Usually the previously executed task will be most active, and selection by the availability heuristic should result in a bias to repeat a task. Research on random number generation has demonstrated a dependence on executive control abilities (Baddeley, Emslie, Kolodny, \& Duncan 1998), further suggesting a relationship between executive control and the selection of tasks in VTS when participants are instructed to perform the tasks in a random sequence.

In VTS, we expected that more-random task choices (i.e., an increase in switch probabilities approaching .5) would be related to higher WMC, because those with higher WMC would be more likely and/or better able to use the representativeness heuristic to guide task choice than those with lower WMC. Those with lower WMC would base their choices on less accurate representations and/or on the availability heuristic and, therefore, would be more likely to repeat a task then those with higher WMC.

Although no study has examined the relationship between individual differences in task choice and WMC, three studies have found evidence that individual differences in mechanisms of cognitive control are related to choice processes. Arrington and Yates (2009) used the Attention Networks Test (ANT; Fan, McCandliss, Sommer, Raz, \& Posner 2002) to measure individual differences in executive control, spatial orienting, and alerting abilities. They found that switch probability was higher for individuals with higher executive control abilities, as measured by the ability to prevent response conflict on a flanker task. Importantly, switch probability was not related to the other attentional mechanisms - spatial orienting and alertingmeasured by the ANT. Inhibition of the previously chosen task has also been implicated as contributing to switch probability in VTS. Individuals who are better able to switch away from the previous trial's task are also less likely to return to the previous trial's task on a subsequent trial (Lien \& Ruthruff, 2008). In addition, Mayr and Bell (2006) observed that more frequent switching is related to smaller switch costs and suggested that individuals better able to inhibit the task completed on the previous trial will have slower repetition trial RTs, thus lowering switch costs, and be better able to switch to a new task, increasing switch probability.

\section{The present experiments}

In two experiments, we examined the relationship between individual differences in WMC as measured by the operation span task (OSPAN; Turner \& Engle, 1989) and measures of task choice and task performance during VTS. We manipulated the response-to-stimulus interval (RSI) in Experiment 1 and examined reliance on environmental support by comparing trials where the stimulus biased a task repetition (i.e., the stimulus repeated) with trials where the stimulus supported a task change (i.e., the stimulus changed). In Experiment 2, we manipulated the potential for interference from an environmental stimulus by using two univalent stimuli on each trial and varying the stimulus onset asynchrony (SOA) between them.

To the degree that the maintenance and updating of task sequences (i.e., for use of the representativeness heuristic) and the inhibition of the previous task set (i.e., for avoiding perseveration through use of the availability heuristic) are needed to make task choices and are reflected in measures of WMC, we predicted that higher WMC would be related to higher switch probability and less dependence on the environment to guide task choice. It was expected that lower WMC would increase reliance on the previously chosen task or the current task environment to guide the current task choice.

Our expectations regarding the relationships of WMC to various task performance measures in VTS were less clear. 
Arguably, switch costs are better indicators of task preparedness in VTS than in standard task-switching methodologies (Demanet et al., 2010), and so may better reflect the role of $\mathrm{WMC}$ in retrieving and maintaining an appropriate task set. In contrast, work by Arrington and Yates (2009) found that executive control measures (as defined by flanker effects in the ANT) were not correlated with switch costs in VTS. To the extent that WMC as measured by the OSPAN task and executive control as measured by the ANT are tapping the same construct, we expected that WMC would not correlate with switch costs.

To preview the results below, our experiments revealed little relationship between task choice measures and WMC. However, consistent relationships of WMC to task performance measures, particularly to switch costs, were observed in both experiments.

\section{Experiment 1}

In Experiment 1, we used a single bivalent stimulus (a digit) and manipulated the RSI (100, 500, 900, and $1,300 \mathrm{~ms})$. It is not clear how variation in WMC might influence switch probability at the different RSIs. On one hand, the relationship might be strongest at the longer RSIs, when there is more time to use the representativeness heuristic to maintain, update, and compare the representations, and thus more time for variation in WMC to be manifest through these processes. On the other hand, to the extent that WMC subserves the inhibition of previously executed tasks or resolution of interference from them, variations in WMC might be critical sooner after the previous task response, at shorter RSIs, when the previous task representation is strongest. Although each of these processes predicts an interaction between WMC and RSI, the combination of them is equally likely and thus may result in overall lower switch probability for individuals with lower WMC with no interaction.

While the VTS paradigm is designed to increase reliance on endogenous mechanisms of task selection by removing explicit cues or sequences of tasks, task selection does not occur in a vacuum, and the external environment may influence task choice. For example, when the stimulus repeats from the previous trial, participants are more likely to repeat the same task than when the stimulus changes (Mayr \& Bell, 2006). This effect is particularly strong when participants are under WM load, suggesting that cognitive control may, in part, shield a selected goal from interference from environmental intrusions (Demanet et al., 2010). Therefore, we expected that the correlations between WMC and mean switch probability would be greater for stimulus repetition trials than for stimulus change trials.
Method

Participants Ninety-four students (49 female; age: $M=$ 19.1 years, $S D=1.6$ ) were recruited from introductory psychology classes at the University of New Mexico and included in the final data set. Twelve individuals were not included in the data set: 4 reported current treatment with psychotropic medications, 5 did not meet the performance criterion for the WM task, 1 had less than $85 \%$ accuracy on the VTS tasks, and 2 switched tasks on fewer than $10 \%$ of trials.

Apparatus and materials VTS materials were presented on a Dell Dimension computer using the E-Prime software package (Schneider, Eschmann, \& Zuccolotto 2002). Stimuli were presented on a 17-in. CRT monitor, and responses were made on a standard QWERTY keyboard. The stimuli were the numbers $1-9$, excluding 5 . The digits appeared in 18-point New Courier font in black on a light gray background. The numbers were $7 \times 5 \mathrm{~mm}$, and viewing distance was not constrained. A small black plus sign appeared at the center of the display throughout the trials, with the target appearing above it.

The OSPAN task was used to measure WMC (Turner \& Engle, 1989). The E-Prime program (version dated 12/2/ 2003) used to administer the task was downloaded from the Engle Attention and Working Memory Lab website, now at www.psychology.gatech.edu/renglelab/Tasks.htm. In this task, each item includes an equation and a word; participants read the equation aloud, verify if the answer given is correct or not, and then read and remember the word that follows the equation for a recall test. In our study, the experimenter controlled the pacing of each item such that the next item was presented immediately after the participant read the to-be-remember word. After reading a series of these items, participants tried to recall all the words from that trial by writing them in the correct order. Each trial varied in length from two to five equation-word pairs. WMC was calculated using the partial-credit unit procedure recommended by Conway et al. (2006). With this procedure, each trial was given a proportion correct score based on the number of words recalled in the correct order. WMC was the average proportion correct across all 12 trials. Individuals with less than $85 \%$ accuracy on the equation verification task were excluded from the data set.

A demographic questionnaire was used that included questions about age, years of education, medications currently being taken, learning disabilities, medical history of stroke or brain trauma, and treatment history for mental illness such as depression.

Procedure On each trial, a randomly selected target digit appeared above the fixation point and remained on screen 
until a response was made. Responses were made with the index and middle fingers of each hand. The parity and magnitude tasks were mapped to different hands, and the stimulus-response mappings were counterbalanced across participants. Following a response the target disappeared, and a fixation point was presented for a variable RSI until the next target appeared. The RSI was manipulated randomly within blocks and was $100,500,900$, or $1,300 \mathrm{~ms}$.

The procedure began with 16 single-task practice trials for the magnitude and parity tasks. Participants then received written and verbal VTS instructions to perform each task equally often and in a random sequence (modeled from Arrington \& Logan, 2004). They completed a block of 64 VTS trials for practice. The experimenter observed the participant's behavior and provided additional instructions if patterns of responding were detected. The experimental trials were presented in 14 blocks of 64 trials. Participants could take self-paced breaks between blocks. The OSPAN task was completed after the VTS procedure and then followed by a demographic questionnaire.

\section{Results}

Trials were sorted into task conditions based on the hand used to make the response for trial $n$, and into task transition conditions (switch vs. repetition) based on the task performed on trials $n$ and $n-1$. Trials were excluded from the switch probability and RT analyses for the following reasons: (1) the first trial of each block, (2) trials with incorrect responses, (3) trials after incorrect responses, and (4) trials with RTs less than 150 or greater than $3,000 \mathrm{~ms}$. For the accuracy analyses, only the first-trial and RT exclusion criteria were used. An alpha level of .05 was used to determine statistical significance. The analyses reported below were also conducted, for both experiments, on measures calculated after excluding trials with a stimulus repetition (12\% of trials). The significant and nonsignificant correlations with WMC were the same in both sets of analyses. We chose to report the analyses with measures including stimulus repetitions.

Error handling excluded $6.1 \%$ of trials from the analyses. RT trimming excluded $1.2 \%$ of the trials. Table 1 presents the means, standard deviations, Cronbach's alpha reliabilities, and WMC correlations for the task choice and switch cost measures.

Task choice Participants showed a small but consistent bias to choose the parity task more often than the magnitude task, with the mean proportion of trials on which the parity task was performed $[p$ (parity)] being significantly larger than $.5, t(93)=4.32$. Further, the correlation of WMC and $p$ (parity) showed that as WMC increased, this bias decreased. Mean switch probability $[p(\mathrm{sw})]$ was significantly less than $.5, t(93)=-8.09$, showing the expected bias. Also, $p(\mathrm{sw})$ differed across RSIs, $F(3,279)=134.10$, $M S E=.002, \eta_{\mathrm{p}}{ }^{2}=.590 . P(\mathrm{sw})$ was not significantly correlated with WMC. Outlier analysis found no participant with $p(\mathrm{sw})$ greater than three standard deviations from the mean. Further exploration of this possible relationship revealed marginally significant $(p<.10)$ correlations of switch probabilities with WMC in the 100- and 1,300-ms RSI conditions (Table 1) that were nominally larger than in the 500- and 900-ms conditions.

In addition, we investigated the relationship of $p(\mathrm{sw})$ to WMC on stimulus repetition trials, where endogenous control of task choice was more difficult because the environment supported a task repetition (Demanet et al., 2010; Mayr \& Bell, 2006). $P($ sw) was lower on stimulus repetition $(M=.347)$ than on stimulus change trials $(M=$ .396), $F(1,93)=28.40, M S E=.016, \eta_{\mathrm{p}}^{2}=.234$, and this difference did not interact with RSI, $F<1$. More importantly, the correlations of WMC with $p(\mathrm{sw})$ on stimulus repetition trials at each RSI and overall were not significant $(r \mathrm{~s}=.147, .087, .063, .155$, and .130 in the 100-, 500-, 900-, and 1,300-ms conditions and overall, respectively), all $p \mathrm{~s}>$ .13. Finally, we calculated the difference between $p$ (sw) for stimulus repetition and stimulus change trials for each RSI and over all trials and correlated these measures with WMC. All of the coefficients were small and not significant $(r \mathrm{~s}=$ -.046, .012, .099, -.007, and .003 in the 100-, 500-, 900-, and 1,300-ms conditions and overall, respectively).

To examine whether the amount of time available to engage in control processes might differ based on WMC, we calculated the change in $p(\mathrm{sw})$ across the levels of RSI, $p(\mathrm{sw})$ slope, for each participant. $P(\mathrm{sw})$ did increase linearly with longer preparation intervals [i.e., $p(\mathrm{sw})$ slope was significantly greater than 0$], t(93)=14.84$. Critically for our hypothesis, this increase in $p(\mathrm{sw})$ with more time for preparation was not correlated with WMC (Table 1).

The proportion of each task performed assesses randomness based on individual trials, and $p(\mathrm{sw})$ assesses randomness from one trial to the next. There are many more measures of whether performance is consistent with a random sequence (for a review, see Towse \& Neil, 1998). To assess randomness across longer sequences of trials and using other algorithms, we used the RGCalc program (Towse \& Neil, 1998). This program calculates measures of redundancy (the degree to which one task was preferred over the other), coupon (the mean number of tasks chosen before both tasks were chosen), runs (the variability in how long a series of different tasks is completed), RNG (how often any one task follows the other task), RNG2 (how often a sequence of the two task choices follows any other sequence of the two task choices), and phi indexes for lags 3-7 (compares frequency of repetitions of tasks at 
Table 1 Experiment 1 means, standard deviations, Cronbach's alpha reliabilities, and correlations with WMC for all trials and excluding stimulus repetition trials

\begin{tabular}{|c|c|c|c|c|c|}
\hline \multirow[b]{2}{*}{ Measures } & \multirow[b]{2}{*}{$M$} & \multirow[b]{2}{*}{$S D$} & \multirow[b]{2}{*}{ Reliabilities } & \multirow{2}{*}{$\frac{\text { All Trials }}{r}$} & \multirow{2}{*}{$\begin{array}{l}\text { Excluding Stimulus Repetitions } \\
r\end{array}$} \\
\hline & & & & & \\
\hline \multicolumn{6}{|l|}{ Task Choice Measures } \\
\hline$p$ (parity) & .511 & .030 & .813 & $-.215^{*}$ & $-.189^{\dagger}$ \\
\hline$p(\mathrm{sw})_{100 \mathrm{RSI}}$ & .319 & .159 & .929 & $.179^{\dagger}$ & $.179^{\dagger}$ \\
\hline$p(\mathrm{sw})_{500 \mathrm{RSI}}$ & .376 & .139 & .926 & .131 & .137 \\
\hline$p(\mathrm{sw})_{900 \mathrm{RSI}}$ & .419 & .129 & .935 & .145 & .165 \\
\hline$p(\mathrm{sw})_{1300 \mathrm{RSI}}$ & .442 & .128 & .955 & $.184^{\dagger}$ & $.190^{\dagger}$ \\
\hline$p(\mathrm{sw})$ slope & .041 & .030 & .734 & -.053 & -.026 \\
\hline \multicolumn{6}{|c|}{ Task Performance Measures } \\
\hline Switch Cost RT & 167 & 79 & .789 & -.146 & -.154 \\
\hline Switch Cost $\mathrm{RT}_{100 \mathrm{RSI}}$ & 287 & 133 & .548 & $-.256^{*}$ & $-.247^{*}$ \\
\hline Switch Cost $\mathrm{RT}_{500 \mathrm{RSI}}$ & 179 & 104 & .554 & -.122 & -.138 \\
\hline Switch Cost $\mathrm{RT}_{800 \mathrm{RSI}}$ & 138 & 94 & .380 & -.043 & -.048 \\
\hline Switch Cost $\mathrm{RT}_{1,300 \mathrm{RSI}}$ & 114 & 93 & .511 & -.094 & -.118 \\
\hline SC slope across RSI & -60 & 38 & .491 & $.179^{\dagger}$ & .165 \\
\hline
\end{tabular}

Note: Cronbach's alpha reliabilities were calculated for each measure across the 14 blocks of the experiment except for the switch cost measures by RSI. For the switch cost reliabilities many participants were missing observations in multiple blocks. Therefore we calculated these reliabilities by comparing the first half of the experiment with the second half of the experiment. $p$ (parity), proportion of times parity task chosen; $p$ (sw), probability of switch. ${ }^{*} p<.05 .^{\dagger} p<.10$

various lags with the expected frequency). Measures of the turning point index and phi index at lag 2 were almost perfectly correlated with $p(\mathrm{sw}), r \mathrm{~s}>.99$, so are not reported. For each participant, each of the 14 blocks of task choices (including error trials) was processed. The average redundancy, coupon, runs, RNG, RNG2, and phi indexes 3-7 measures were computed across blocks for each participant and then correlated with WMC. WMC was negatively correlated with the redundancy measure, $r=$ -.250 , indicating that as $\mathrm{WMC}$ increased individuals were better able to do each task equally often. This finding is consistent with the correlation of $p$ (parity) with WMC. None of the other randomness measures were significantly correlated with WMC (coupon, $r=-.148$; runs, $r=-.074$; RNG, $r=-.187$; RNG2, $r=-.006$; and phi indexes 3-7, $r \mathrm{~s}=-.091,-.134, .044,-.108$, and -.020 , respectively; all $p$ s $>.07)$. Finally, the mean number of repetitions in a sequence was 2.8 trials $(S D=1.0)$, and this measure did not correlate with WMC, $r=-.19$.

Task performance Mean RTs are available in Table 2 and were submitted to a 4 (RSI) $\times 2$ (transition type) withinsubjects ANOVA. Significant switch costs were observed; switch trials were slower $(M=972 \mathrm{~ms})$ than repetition trials $(M=797 \mathrm{~ms}), F(1,93)=476.76, M S E=12,209, \eta_{\mathrm{p}}{ }^{2}=$ .837. RTs were longer at the shortest RSIs than at the longer intervals $(M \mathrm{~s}=937,882,857$, and $862 \mathrm{~ms}$ for the 100-, 500-, 900-, and 1,300-ms RSIs, respectively), $F(1,93)=$
71.73, MSE $=3,554, \eta_{\mathrm{p}}^{2}=.435 . \mathrm{RT}$ switch costs decreased as RSI increased as a result of faster switch trial RTs with increasing RSI (see Table 2), $F(1,93)=118.21, M S E=$ $2577, \eta_{\mathrm{p}}{ }^{2}=.560$. Paralleling the RT analysis, accuracy was higher on repetition $(M=.971)$ than on switch trials $(M=$ $.967), F(1,93)=5.31, M S E=.001, \eta_{\mathrm{p}}^{2}=.054$. Accuracy also varied slightly in a nonmonotonic pattern as a function of RSI $(M \mathrm{~s}=.971, .969, .965$, and .970 for the 100-, 500-, 900-, and 1,300-ms RSI conditions, respectively), $F(1,93)=$ 3.94, MSE $<.001, \eta_{\mathrm{p}}{ }^{2}=.041$. Accuracy switch costs did not vary by RSI, $F(3,279)=1.40, M S E<.001, \eta_{\mathrm{p}}{ }^{2}=.015$.

WMC was not related to RTs $(M=795, S D=116, r=$ .113 , for repetition trials; $M=963, S D=164, r=.001$, for switch trials), but higher WMC scores were accompanied by more accurate performance on both repetition $(M=$ $.971, S D=.02, r=.297, p<.05)$ and switch $(M=.967$, $S D=.03, r=.246, p<.05)$ trials. As can be seen in Table 1, WMC had a significant negative correlation with RT switch costs, but only at the shortest RSI. Scatterplots of switch costs by WMC for each RSI condition are presented in Appendix A, Fig. 1. In addition, no outliers were found in the data; in other words, all participants' average switch costs in the 100-ms RSI condition were within three standard deviations of the mean. Switch costs computed from the accuracy measures were not correlated with WMC at any RSI or overall $(r \mathrm{~s}=.048, .025,-.075,-.031$, and -.007 for the $100-, 500-, 900-$, and $1,300-m s$ conditions and overall, respectively). 
Table 2 Mean RTs and accuracies by RSI and transition type in Experiment 1

\begin{tabular}{|c|c|c|c|c|c|c|c|c|c|}
\hline \multirow[b]{3}{*}{ Dependent Variables } & \multirow[b]{3}{*}{ Condition } & \multicolumn{8}{|l|}{ RSI } \\
\hline & & \multicolumn{2}{|c|}{$100 \mathrm{~ms}$} & \multicolumn{2}{|c|}{$500 \mathrm{~ms}$} & \multicolumn{2}{|c|}{$900 \mathrm{~ms}$} & \multicolumn{2}{|c|}{$1,300 \mathrm{~ms}$} \\
\hline & & $M$ & $S E$ & M & $S E$ & $M$ & $S E$ & $M$ & $S E$ \\
\hline \multirow[t]{3}{*}{ RTs (ms) } & Repetitions & 794 & 12 & 792 & 13 & 788 & 12 & 812 & 14 \\
\hline & Switches & 1,081 & 18 & 971 & 17 & 926 & 17 & 912 & 17 \\
\hline & Switch Cost & 287 & & 179 & & 138 & & 100 & \\
\hline \multirow[t]{3}{*}{ Accuracy } & Repetitions & .976 & .002 & .971 & .003 & .966 & .003 & .971 & .003 \\
\hline & Switches & .967 & .004 & .966 & .004 & .964 & .003 & .969 & .003 \\
\hline & Switch Cost & \multicolumn{2}{|l|}{-.009} & \multicolumn{2}{|l|}{-.004} & \multicolumn{2}{|l|}{-.002} & \multicolumn{2}{|l|}{-.002} \\
\hline
\end{tabular}

\section{Discussion}

Consistent with previous work, a robust repetition biasthat is, to switch less often than true randomness would dictate-was observed (e.g., Arrington \& Logan, 2005), and this bias was more pronounced when the current trial stimulus was a repetition of the previous stimulus than when it was a new stimulus (Demanet et al., 2010). $P(\mathrm{sw})$ was not significantly correlated with WMC, though, regardless of whether the stimulus repeated or changed. Further, the degree to which an individual was more likely to rely on the environment to guide task choice [i.e., the difference between $p(\mathrm{sw})$ for stimulus repetition vs. stimulus change trials] was not related to WMC. With a sample of 94 participants, the failure of the correlations of WMC with $p(\mathrm{sw})$ to reach significance suggests that the relationship of random-choice processes with $\mathrm{WMC}$, if it exists, is small and fragile (Cohen, 1988). In Experiment 2, we used a stronger manipulation of environmental stimuli to guide task choice in order to further explore this issue.

Turning to the task performance measures, higher WMC was related to smaller switch costs at the fastest RSI. Further, RTs were not significantly related to WMC, ruling out the possibility that larger switch costs for those low in WMC were the result of slower RTs on all trials. These task performance measures may reflect establishing the appropriate control parameters for completion of the chosen task, such as preparing attentional control settings (Arrington, Altmann, \& Carr 2003) and/or retrieving representations of task rules or stimulus-response mappings (Mayr \& Kliegl, 2000).

Lower WMC was associated with more frequent (and less equal) production of the parity task. This finding suggests that lower WMC may be related to an inability to keep track of how often each task has been completed and greater susceptibility to reliance on small differences in task difficulty (see, e.g., Demanet et al., 2010; Yeung, 2010). Task difficulty may have been induced by the order in which tasks were practiced; the parity task was the first task participants practiced. The initial task performed by a participant during the experimental session has been shown to bias later task choices (Arrington, Weaver, \& Pauker 2010). The correlation of $p$ (parity) with WMC suggests that this bias was more pronounced for individuals with lower WMC.

\section{Experiment 2}

One criticism of Experiment 1 is that the benefit of using exogenous control processes to guide random task choice may not have been sufficient to induce reliance on such processes because the bivalent stimuli supported the endogenous control of choice and/or because stimuli repeated on only $12 \%$ of trials. Previous research has shown that WMC-related differences in performance emerge when exogenous control processes are sufficient for task performance in most conditions and endogenous control mechanisms only optimize performance or are required infrequently. An example comes from the study of visual search performance. One study using standard visual search paradigms found that when the most efficient visual search could be achieved by using the perceptual properties of the search array to guide search in a bottomup fashion, no relationship was observed with WMC (Kane, Poole, Tuholski, \& Engle 2006). However, in a different study, when more efficient search could be achieved by using knowledge about the contents of the search arrays in a top-down manner to control the search, WMC differences were observed (Sobel, Gerrie, Poole, \& Kane 2007). Another example comes from examining the relationship of WMC to Stroop task performance (Kane \& Engle, 2003). When endogenous control was required on the majority of trials (i.e., conditions with a high proportion of incongruent trials), WMC-related differences on incongruent trials were quite small. However, when outsourcing control to the environment is sufficient for accurate performance on a majority of trials 
(i.e., conditions with a high proportion of congruent trials), WMC-related differences were larger. In Experiment 1, the benefit of using exogenous control processes associated with stimulus repetitions to guide task choice might not have been sufficient to induce reliance on them because the stimuli repeated on only $12 \%$ of trials. Therefore, we thought that it was important to include a manipulation in the VTS task that would often allow participants to use exogenous control to guide task choice, even if that strategy was not optimal.

In Experiment 2, we thus adopted a manipulation that allowed information in the environment to guide task choice on some trials through variation in stimulus availability (Arrington, 2008). In this procedure, each target array consists of two stimuli, one for each of the possible tasks. The stimuli could be presented at different times through a manipulation of SOA. This procedure introduced a further task choice measure, the probability of choosing the task associated with the first stimulus, $p(\mathrm{~S} 1)$. At nonzero SOAs (e.g., 50, 100, and $150 \mathrm{~ms}$ ), $p(\mathrm{~S} 1)$ is greater than would be expected by chance (i.e., >.5) and increases as SOA increases (Arrington, 2008). Thus, exogenous control based on the identity of $\mathrm{S} 1$ is used to guide task choice on some, but not all, trials. Further, $p(\mathrm{~S} 1)$ decreases with longer preparation intervals, suggesting that the influence of stimulus availability on task choice may compete with the goal of using endogenous control (e.g., representativeness or availability heuristics) to guide behavior. Endogenous control is still required on trials with a 0-ms SOA, however, and participants are still instructed to choose the task to perform on each trial in a random order, as if flipping a coin.

Following Arrington (2008), we used target arrays with two stimuli (a letter and a number) and manipulated the availability of one stimulus prior to the other by varying $\operatorname{SOA}(0,50,100$, and $150 \mathrm{~ms})$ such that one stimulus was available for task choice prior to the other on $75 \%$ of trials. The stimulus availability manipulation might increase reliance on the environment to guide task choice as well as the potential for neglect of the goal to choose tasks according to endogenous control processes. Because S1 is determined randomly, a seemingly random choice can be achieved on $75 \%$ of trials (i.e., those with the nonzero SOAs) by using exogenous control (based on S1 availability) to guide task selection. Consistent with the view that WMC is related to the ability to use endogenous control processes for task choice even when reliance on exogenous control is sufficient in many cases, we would expect WMC to be related to the probability of choosing the task associated with the first stimulus, $p(\mathrm{~S} 1)$. Specifically, we expected lower WMC to be related to a greater reliance on exogenous control that would lead to higher $p(\mathrm{~S} 1)$. In addition, we predicted that on $0-\mathrm{ms}$ SOA trials, because those with low WMC are not maintaining the goal of being random, they would be more likely to repeat the task just completed on the previous trials than those with high WMC.

\section{Method}

Participants Eighty-five students (54 female; age, $M=$ 19.5 years, $S D=2.2$; WMC score $=.73, S D=.01)$ from introductory psychology courses at the University of New Mexico were included in the final data set. Four participants were excluded because of invalid WMC task performance: 2 delayed equation verification to rehearse the words, and 2 did not meet the accuracy criterion. Seven participants were excluded because of VTS task performance: 4 had less than $85 \%$ accuracy on the VTS tasks, and 3 switched tasks on less than $10 \%$ of trials.

Apparatus and materials The apparatus and materials were the same as in Experiment 1 except for the following: The stimuli were the numbers 2-9 and the letters A, E, I, U, B, $\mathrm{C}, \mathrm{L}$, and $\mathrm{W}$. The fixation screen had a plus sign in the center of the display and four pound signs (\#) as placeholders marking possible target locations. Four potential target locations were used in order to avoid participants relying on a strategy of selecting the task to perform based on the location where the target appeared. The target locations were positioned $15 \mathrm{~mm}$ from fixation to the edge of the stimulus along the diagonals. All stimuli were presented in black on a light gray background. The placeholders and target stimuli were $7 \times 5 \mathrm{~mm}$, printed in Courier New font type.

Procedure Each trial began with the fixation screen displayed for 500 or 1,300 ms. The RSI was chosen randomly. Then two target stimuli, a digit and a letter, replaced the placeholders in two of the four possible target locations. The target stimuli appeared with SOAs of 0,50 , 100 , or $150 \mathrm{~ms}$. The SOA and location of the targets were selected at random. The identity of the first stimulus to appear was selected at random, with the letter and number occurring as the first stimulus equally often. Participants were asked to make a parity judgment on the digit or a consonant/vowel judgment on the letter by pressing the appropriate keys. Following a response, the fixation screen was immediately presented. The stimulus-response mappings were counterbalanced across participants, with each task being mapped to the index and middle fingers of both hands.

Participants began the procedure with 16 trials of singletask practice for both the number and letter tasks. Then they received the VTS instructions to perform each task equally 
often and in a random sequence (modeled from Arrington \& Logan, 2004). Participants practiced task switching for 32 trials. Then data collection occurred over 14 blocks of 64 trials, with self-paced breaks between trials. As in Experiment 1, following the VTS procedure, all participants completed the OSPAN task. Finally, participants completed the demographic questionnaire.

\section{Results}

Tasks and task transitions were coded in the same manner as in the previous experiment. On trials with a $0-\mathrm{ms} \mathrm{SOA}$, the "S1" was designated randomly.

Error handling excluded $7.8 \%$ of trials from the analyses, and RT trimming excluded $1.7 \%$ of the trials. Summary statistics for measures of task choice and task performance, along with reliabilities and correlations between WMC, as measured by the OSPAN, and these measures, are provided in Table 3.

Task choice The mean proportion of trials on which the letter task was performed was .507; although this was numerically similar to .5 , it was significantly greater, $t(84)=2.51$. The data suggest that overall, participants were able to perform the two tasks generally equally often. WMC did not correlate with the proportion of letter task trials.

The effect of stimulus availability on task choice replicated previous findings, with the $p(\mathrm{~S} 1)$ increasing .03 for each level of SOA, a slope significantly different from $0, t(84)=10.34[p(\mathrm{~S} 1) M \mathrm{~s}=.499, .536, .567$, and .602 for the $0-, 50-, 100-$, and 150-ms SOAs, respectively], $F(3$, $252)=63.24, M S E=.005, \eta_{\mathrm{p}}{ }^{2}=.429$. However, RSI did not influence $p(\mathrm{~S} 1), F(1,84)=1.29, M S E=.003, \eta_{\mathrm{p}}{ }^{2}=$ .015 , and did not interact with SOA, $F<1$.

To examine the influence of WMC on the utilization of stimulus availability in task choice, we correlated WMC with the average $p(\mathrm{~S} 1)$ across the three nonzero SOA conditions. Scatterplots of $p(\mathrm{~S} 1)$ by WMC for each RSI condition are presented in Appendix A, Fig. 2. In addition, the change in $p(\mathrm{~S} 1)$ across the four levels of SOA was calculated as a slope, $p(\mathrm{~S} 1)$ slope (see Table 3). Neither the correlation of WMC with average $p(\mathrm{~S} 1)$ across the 50-, $100-$, and $150-\mathrm{ms}$ SOA conditions nor the correlation of $\mathrm{WMC}$ and $p(\mathrm{~S} 1)$ slope was significant. The $r$ values suggested that a small relationship might be found with a larger sample size (see Table 3; Cohen, 1988). Importantly, when the data were investigated for outliers, the exclusion of the 1 participant with a $p(\mathrm{~S} 1)$ slope greater than three standard deviations from the mean reduced the coefficients substantially, $p(\mathrm{~S} 1) r=.071$ versus $p(\mathrm{~S} 1)$ slope $r=.034$, suggesting that any relationship between WMC and stimulus availability was not robust.

Reliance on stimulus availability at nonzero SOAs necessarily elevates the $p(\mathrm{sw})$ measure, so we report the analyses of $p(\mathrm{sw})$ only in the 0 -ms SOA condition. ${ }^{1}$ The patterns of significance were the same when all SOA conditions were included. Switch probability $(M=.384)$ was significantly less than $.5, t(84)=-8.23$, showing the expected bias. In addition, the bias decreased as RSI increased; switching was less likely at the 500-ms RSI than at the 1,300 -ms RSI, $F(1,84)=68.06, M S E=.002$, $\eta_{\mathrm{p}}{ }^{2}=.448$. Neither the 500-ms nor the 1,300-ms switch probability, nor the reduction in switch probabilities across RSIs, correlated with WMC (see Table 3).

Following the same procedure as described for Experiment 1, we used the RGCalc program (Towse \& Neil, 1998) to compute additional measures of randomness. None of the measures (redundancy, $r=.011$; coupon, $r=-.040$; runs, $r=-.049$; RNG, $r=.002$; $\mathrm{RNG} 2, r=.001$; and phi indexes $3-7, r \mathrm{~s}=-.064,-.109$, $-.043,-.006$, and -.076 , respectively; all $p$ s $>.32$ ) were related to WMC. Finally, the mean number of task repetitions in a sequence was $2.3(S D=0.59)$ trials, and this measure did not correlate with WMC, $r=.02$.

Task performance Mean RTs were submitted to a $4(\mathrm{SOA}) \times$ 2 (RSI) $\times 2$ (transition) within-subjects ANOVA and are presented in Table 4. Switch trials were slower $(M=$ $1,089 \mathrm{~ms})$ than repetition trials $(M=930 \mathrm{~ms}), F(1,84)=$ 206.36, $M S E=41,486, \eta_{\mathrm{p}}{ }^{2}=.711$. There was not a main effect of RSI, $F<1$, but there was clear evidence for a reduction in switch costs with increasing RSI. The difference between switch and repetition trial RTs was greater in the 500-ms RSI condition $(M \mathrm{~s}=1,107$ and $915 \mathrm{~ms}$, respectively) than in the 1,300-ms RSI condition $(M \mathrm{~s}=1,072$ and $946 \mathrm{~ms}$, respectively) according to a significant Transition $\times$ RSI interaction, $F(1,84)=83.77$, $M S E=4,427, \eta_{\mathrm{p}}{ }^{2}=.499$. RTs were slower when both stimuli were presented at the same time $(0-\mathrm{ms} \mathrm{SOA})$ than at the longer SOAs $(M \mathrm{~s}=1,021,1,008,1,003$, and $1,007 \mathrm{~ms}$, respectively, for the $0-, 50-, 100-$, and $150-\mathrm{ms}$ SOAs), $F(3,252)=4.53, M S E=4,387, \eta_{\mathrm{p}}{ }^{2}=.051$. SOA did not enter into any interactions with RSI or transition [SOA $\times$ RSI: $F(3,252)=1.26, M S E=3,861, \eta_{\mathrm{p}}{ }^{2}=.015$; SOA $\times$ Transition: $F<1$; SOA $\times$ RSI $\times$ Transition: $F(3$, $\left.252)=1.34, M S E=4,221, \eta_{\mathrm{p}}^{2}=.0156\right]$.

\footnotetext{
${ }^{1}$ Switch probability was not analyzed as a function of the SOA because it is not clear how to interpret this manipulation for the switch probability measure. The letter and number stimuli were assigned randomly to be S1 and S2 on each trial. Therefore, changes in the probability with SOA might be due to the SOA manipulation or to the random assignment of stimuli to the S1 position.
} 
Table 3 Experiment 2 means, standard deviations, Cronbach's alpha reliabilities, and correlations with WMC for all trials and when stimulus repetition trials were excluded

\begin{tabular}{|c|c|c|c|c|c|}
\hline \multirow[b]{2}{*}{ Measures } & \multirow[b]{2}{*}{$M$} & \multirow[b]{2}{*}{$S D$} & \multirow[b]{2}{*}{ Reliabilities } & \multirow{2}{*}{$\frac{\text { All Trials }}{r}$} & \multirow{2}{*}{$\begin{array}{l}\text { Excluding Stimulus Repetitions } \\
r\end{array}$} \\
\hline & & & & & \\
\hline \multicolumn{6}{|l|}{ Task Choice Measures } \\
\hline$p$ (letter task) & .507 & .027 & .755 & .132 & .071 \\
\hline$p(\mathrm{sw})_{500 \mathrm{RSI} 0 \text {-msSOA }}$ & .369 & .124 & .952 & -.008 & -.014 \\
\hline$p(\mathrm{sW})_{1,300 \mathrm{RSI} 0 \text {-msSOA }}$ & .422 & .114 & .945 & .016 & -.021 \\
\hline Change in $p(\mathrm{sw})$ from 500 to $1,300 \mathrm{~ms}$ RSI & .054 & .039 & .292 & .077 & .062 \\
\hline$p(\mathrm{~S} 1)$ & .568 & .068 & .866 & $.189^{\dagger}$ & .159 \\
\hline$p(\mathrm{~S} 1)$ slope & .034 & .030 & .725 & .140 & .158 \\
\hline \multicolumn{6}{|l|}{ Task Performance Measures } \\
\hline Switch Cost $\mathrm{RT}_{500 \mathrm{RSI}}$ & 203 & 121 & .812 & $-.214 *$ & $-.226^{*}$ \\
\hline Switch Cost $\mathrm{RT}_{1,300 \mathrm{RSI}}$ & 133 & 98 & .733 & -.083 & -.163 \\
\hline Reduction in Switch Cost RT across RSI & 66 & 67 & .046 & -.161 & $-.192^{\dagger}$ \\
\hline
\end{tabular}

Note: Switch probabilities are for the 0 -ms SOA condition only. Cronbach's alpha reliabilities were calculated for each measure across the 14 blocks of the experiment. For the switch cost reliability measures, six individuals that did not have observations in some blocks were excluded from the reliability analysis. $p$ (letter), proportion of times letter task chosen; $p$ (sw), probability of switch. $*^{*} p<.05 .^{\dagger} p<.10$

Higher WMC scores were accompanied by faster RTs on both repetition $(r=-.371)$ and switch $(r=-.412)$ trials. Scatterplots of RT switch costs by WMC for each RSI condition are presented in Appendix A, Fig. 1. When only $500 \mathrm{~ms}$ was available to prepare for the upcoming task, there was a significant negative correlation between WMC and switch costs, but not when more time $(1,300 \mathrm{~ms})$ was available. This pattern resulted in a nonsignificant correla- tion of -.161 between WMC and the reduction in RT switch costs across the RSIs.

Paralleling the RT analyses, repetition trials were more accurate $(M=.964, S D=.023)$ than switch trials $(M=.956$, $S D=.041), F(1,84)=10.04, M S E=.002, \eta_{\mathrm{p}}^{2}=.107$. The main effect of RSI was not significant, $F<1$, but RSI did interact with transition such that switch costs were smaller at the $1,300-\mathrm{ms} \operatorname{RSI}(M=.004)$ than at the 500 -ms RSI

Table 4 Mean RTs and accuracies by SOA, RSI, and transition type in Experiment 2

\begin{tabular}{|c|c|c|c|c|c|c|c|c|c|}
\hline \multirow[b]{3}{*}{ Dependent Variables } & \multirow[b]{3}{*}{ Condition } & \multicolumn{8}{|l|}{ SOA } \\
\hline & & \multicolumn{2}{|l|}{$0 \mathrm{~ms}$} & \multicolumn{2}{|l|}{$50 \mathrm{~ms}$} & \multicolumn{2}{|c|}{$100 \mathrm{~ms}$} & \multicolumn{2}{|c|}{$150 \mathrm{~ms}$} \\
\hline & & $M$ & $S E$ & $M$ & $S E$ & $M$ & $S E$ & $M$ & $S E$ \\
\hline \multicolumn{10}{|l|}{ 500-ms RSI } \\
\hline \multirow[t]{3}{*}{ RTs (ms) } & Repetitions & 921 & 15 & 917 & 15 & 910 & 17 & 911 & 16 \\
\hline & Switches & 1,119 & 20 & 1,095 & 21 & 1,097 & 20 & 1,115 & 21 \\
\hline & Switch Cost & 198 & & 178 & & 187 & & 203 & \\
\hline \multirow[t]{3}{*}{ Accuracy } & Repetitions & .969 & .003 & .967 & .003 & .967 & .003 & .964 & .004 \\
\hline & Switches & .954 & .007 & .953 & .005 & .953 & .006 & .956 & .006 \\
\hline & Switch Cost & -.015 & & -.014 & & -.014 & & -.008 & \\
\hline \multicolumn{10}{|l|}{ 1,300-ms RSI } \\
\hline \multirow[t]{3}{*}{ RTs } & Repetitions & 958 & 17 & 944 & 17 & 943 & 17 & 940 & 17 \\
\hline & Switches & 1,085 & 22 & 1,078 & 20 & 1,063 & 20 & 1,061 & 18 \\
\hline & Switch Cost & 127 & & 135 & & 120 & & 121 & \\
\hline \multirow[t]{3}{*}{ Accuracy } & Repetitions & .959 & .004 & .964 & .003 & .959 & .004 & .959 & .004 \\
\hline & Switches & .961 & .005 & .958 & .005 & .956 & .006 & .954 & .005 \\
\hline & Switch Cost & .002 & & -.007 & & -.003 & & -.005 & \\
\hline
\end{tabular}


$(M=.013), F(1,84)=10.12, M S E=.001, \eta_{\mathrm{p}}{ }^{2}=.107$. No other effects or interactions were significant, $F_{\mathrm{S}}<1$.

Switch $(r=.007)$ and repetition $(r=.066)$ trial accuracy did not differ with WMC. Switch costs in the accuracy measure were not correlated with WMC at either RSI $(r=$ .007 for the $500-\mathrm{ms}$ and $r=-.113$ for the $1,300-\mathrm{ms}$ RSI condition).

\section{Discussion}

This experiment introduced a stimulus availability manipulation to increase the likelihood that individuals with low WMC might neglect the goal of choosing tasks to be performed and instead outsource control to the environment and execute the task associated with the first available stimulus. However, as in Experiment 1, none of the measures of task choice, $p(\mathrm{sw}), p(\mathrm{~S} 1)$, or $p(\mathrm{~S} 1)$ slope were significantly related to WMC. Switch costs in the fastest RSI condition were related to WMC, though.

Replicating Arrington (2008), we found that task choice was influenced by stimulus availability; however, even at the longest SOAs task choice was not based exclusively on availability [i.e., the $p(\mathrm{~S} 1)$ value was only .602], suggesting that the effect of external stimuli accounted for a reliable but small proportion of the variance in task choice and that endogenous control processes also accounted for part of the variability. Further, as the SOA increased, in both experiments, stimulus availability had a larger effect, suggesting variation in whether availability information was used to guide task choice, and thus, variation in the reliance on exogenous control. Based on these findings, it appears that the current task environment likely creates a situation in which control processes may be required to limit the influence of stimulus availability information on task choice processes. However, we found no correlations between the measures of the influence of stimulus availability and WMC.

Two of the primary measures of interest, switch probability and switch costs, replicated the effects in Experiment 1. Switch probability in the 0-ms SOA condition again showed the basic repetition bias, and in addition, this bias decreased as the preparation interval increased. Differences in WMC once again did not influence the final task transition choices, though. In terms of task performance, higher WMC was related to smaller switch costs at the shortest preparation interval $(500 \mathrm{~ms})$. The implication of this relationship will be further discussed below.

It is important to note that the results of this experiment suggest an alternative to the interpretation originally proposed by Arrington (2008) of the processing effects of the stimulus availability manipulation. In that work, it was suggested that the utilization of endogenous control through availability and representativeness heuristics should prevent the influence of stimulus availability on task choice. There are two reasons to suspect that our participants were not utilizing the preparation interval (engaging endogenous control) to avoid the influence of the first stimulus on task choice and, in fact, were occasionally strategically using S1 information to guide task choice. First, unlike Arrington, we did not find that the influence of the first stimulus was modulated by the preparation interval. Our preparation intervals $(500$ and $1,300 \mathrm{~ms})$ were different from Arrington's (400 and 2,000 ms), so perhaps the additional time Arrington provided in the long preparation interval condition (700 ms greater than our long RSI) was enough to allow for more complete preparation of task sets, limiting the influence of the available stimulus on task choice. At longer preparation intervals, perhaps we would see evidence for prevention of the influence of stimulus availability (although, in the cued task-switching literature, $1,300 \mathrm{~ms}$ is argued to be sufficient time for preparation; see, e.g., Monsell, 2003). Second, greater utilization of endogenous control should both lead to an avoidance of choosing the $\mathrm{S} 1$ stimulus [lower $p(\mathrm{~S} 1)$ ] and greater switching [larger $p(\mathrm{sw})$ in the 0 -ms SOA condition]. Rather than the expected negative correlation between $p(\mathrm{sw})$ and $p(\mathrm{~S} 1)$, we observed a positive correlation, $r=.386$. This suggests that the same processes that improved random switching (i.e., endogenous control) were also used to choose the S1 task on some trials. Because the experiment was designed such that the first stimulus itself was chosen randomly from either task, using that information to guide task selection would lead to random behavior and also might not tax limited choice processing. However, delaying task choice until stimulus onset can have the beneficial effect of reducing the availability of the previous task set, either actively or passively. In turn, the reduced availability of the previous task set could increase switch probability through more reliance on the representativeness heuristic and/or stimulus availability.

Taken together, this evidence suggests that the stimulus availability manipulation did introduce conditions that promoted using various strategies for achieving random behavior in task choice, including reliance on exogenous control on many, but not all (0-ms SOA and the $40 \%$ of trials where S1 was not chosen), trials. However, even if participants were not trying to resist the influence of exogenous control, performance did indicate that they were moving between strategies of basing control on endogenous (previous task availability) and exogenous (current-trial stimulus availability) control. Importantly, this allocation of attentional resources appears to have operated similarly regardless of WMC. 


\section{General discussion}

In two experiments, participants completed a VTS procedure, choosing which of two tasks would be performed on each trial. To examine how individual differences in WMC influence the interplay of endogenous and exogenous control processes in determining task choice and task performance, we examined the influences of stimulus repetition (Experiment 1 ) and stimulus availability by varying the stimulus onsets within a trial (SOA, Experiment 2). To examine how different endogenous control processes may be utilized based on available WMC, we varied the preparation interval (RSI, Experiments 1 and 2). The most robust relationship to WMC was found for task performance measures. WMC was related to RT switch costs in both experiments, but only at the fastest RSIs (100 and $500 \mathrm{ms,}$ respectively).

In contrast, there were no significant correlations between WMC and either the probability of switching between tasks or the likelihood of selecting a task based on stimulus availability. WMC was not related to the likelihood that the environment would be used to guide task choice rather than endogenous control processes. In Experiment 1, the likelihood of repeating a task when the stimulus repeated was unrelated to WMC. Likewise, the influence of stimulus availability on task choice was not related to WMC when a single outlier was removed, and even with the outlier the correlations did not reach significance. These findings are further discussed below. In neither experiment was WMC related to the endogenous control processes that support random task choice [i.e., $p(\mathrm{sw})$ and other randomness measures]. WMC was related to the ability of participants to do each task equally often (Experiment 1), a finding that was not replicated in Experiment 2. Perhaps those with higher WMC were better able to keep track of how many trials of each task had been completed in Experiment 1, but because of this influence of the stimulus availability on task choice, keeping track of the tasks completed was not as important in Experiment 2.

\section{Task performance}

To our knowledge, the correlations of WMC and switch costs at the fastest RSIs in Experiment $1(100 \mathrm{~ms})$ and Experiment $2(500 \mathrm{~ms})$ are the first demonstration of this relationship (for negative findings, see Kane et al., 2007; Miyake et al., 2000; Oberauer et al., 2000, 2003). Under conditions that varied the RSI (Experiment 1: 100, 500, 900, and 1,300 ms; Experiment 2: 500 and 1,300 ms), individuals with higher WMC were less slowed by the demands of switching tasks than were individuals with lower WMC, but only at the fastest RSIs. This relationship may be due to the role WMC plays in regulating the influence of previously activated stimulus-response rules on the current trial, either through inhibition of the previous task rules or through robust representation of the present stimulus-response rules. The inhibition explanation does not do a good job accounting for our data, though. Specifically, poor inhibition of the previous tasks' response rules should lead to interference (slower RTs) on switch trials and facilitation (faster RTs) on repetition trials. If inhibition processes are the critical mechanism indexed by WMC, we would expect a negative correlation between WMC and switch trial RTs, but a positive correlation between WMC and repetition trial RTs. Contrary to this prediction, in Experiment 1 the correlations were not significant $(r \mathrm{~s}=.136$ for switch and -.074 for repeat trials), and in Experiment 2 both of the correlations were significant and negative $(r \mathrm{~s}=-.427$ and -.382 , respectively).

The relationship between WMC and switch costs is more consistent with the proposal that WMC is related to the ability to retrieve representations from long-term memory and robustly represent them (Unsworth \& Engle, 2007). Why should this relationship be observed at the fastest RSIs (100 and $500 \mathrm{~ms}$, respectively) relative to the other RSIs in each experiment, though, rather than at the same absolute RSIs (e.g., the 500-ms condition in Experiment 1)? Certainly, one possibility is that the different procedures used in the two experiments influenced retrieval speeds differently. Notably, RTs were slower in Experiment 2 than in Experiment 1. Another possibility is that the retrieval of rule representations is sensitive to the retention interval ratio. ${ }^{2}$ This question is one for future research. In VTS, when little time is available between trials (100 or $500 \mathrm{~ms})$, slower retrieval of the stimulus-response rules for a new task and/or poorer representations of those rules (perhaps through weaker associations between the stimuli and responses) reveal the role of WMC in task preparation (see also Heitz \& Engle, 2007). Representational difficulties related to WMC can also account for the relationship of accuracy (Experiment 1) and RTs (Experiment 2) to WMC. Lower WMC was related to poorer establishment or maintenance of the task rules, regardless of whether the task repeated or switched.

Previous attempts to observe the effects of WMC on task preparation processes may have been plagued by the impurity of the switch cost measure. For example, switch costs index not only task performance processes, but also cue encoding (see, e.g., Arrington, Logan, \& Schneider 2007; Logan \& Bundesen, 2003). The present results raise an interesting alternative, though. Both of our experiments varied the RSI. Previous attempts to correlate switch costs with WMC in cued task switching did not appear to examine the relationship of WMC to switch costs at

\footnotetext{
${ }^{2}$ We thank Michael Kane for this suggestion.
} 
different RSIs (Kane et al., 2007; Miyake et al., 2000; Oberauer et al., 2000, 2003). Kane et al. did not report any within-subjects manipulations, and the remaining studies measured switch costs by aggregating across multiple taskswitching measures in which RSI either was not manipulated (because the Jersild list procedure was used; Miyake et al., 2000) or was held constant (Oberauer et al., 2000, 2003, used a single short RSI of either 300 or $400 \mathrm{~ms}$ ). Predictable preparation intervals cause participants to delay task preparation until stimulus onset on a larger proportion of trials (Altmann, 2004). It is possible that in a cued taskswitching study with varying preparation intervals, WMC might influence task performance processes at short RSIs.

A reason to disfavor the possibility that cued taskswitching procedures might produce switch costs that correlate with WMC if the preparation interval is manipulated comes from a recent study by Liefooghe et al. (2009). They found that in a cued task-switching procedure any reductions in switch costs with RSI were not consistent for within- and between-subjects RSI manipulations, but the relationship between RSI and switch costs was robust in the VTS procedure. These findings are consistent with the previous suggestion that even under varying RSIs, switch cost measures in cued task-switching procedures may be a mixture of task reconfiguration and interference resolution processes (e.g., Vandierendonck et al., 2010), some of which are sensitive to WMC and some of which are not. Thus, switch cost measures in these paradigms may be more difficult to relate to WMC.

An influence of WMC on task performance was also supported by the correlations with accuracies in Experiment 1 and RTs in Experiment 2. It is not clear why the pattern varied across experiments, but it may reflect the different procedures used. The correlations provide additional evidence that WMC played a role in behavior, but it is not clear what the source of this difference was. Less efficient task choice processes related to lower WMC may have slowed responses (Experiment 2). Indeed, RTs are positively related to $p(\mathrm{sw})$ (Arrington \& Yates, 2009). However, it is not clear how less efficient task choice would lead to less accurate responses (Experiment 1), except if we assume that task choice and response rule representation fail completely. Therefore, we prefer the interpretation that these WMC differences are related to the robustness of retrieving and maintaining response rule representations during task performance.

\section{Task choice}

We predicted that WMC would be related to switch probability more when the stimuli repeated than when they switched, and more when task choices on many trials could be made based on stimulus availability. These predictions were generated from the view that task choice processes include maintaining a representation of the most recent sequence of tasks, updating that representation as new tasks are performed, and making a choice about what constitutes a random sequence while resisting the biasing influence of the previously executed task (Arrington \& Logan, 2005; Mayr \& Bell, 2006) and while ignoring environmental information to guide task choice (Arrington, 2008; Arrington \& Logan, 2005; Arrington et al., 2010; Demanet et al., 2010). Goal maintenance and updating while resisting the biasing influence of competing sources of information seem to be precisely the conditions that reveal WMC-related differences in other tasks (see Kane et al., 2007, for a review). Further, executive control as measured through a flanker manipulation in the ANT is related to switch probability (Arrington \& Yates, 2009). However, the relationship between WMC and task choice was not observed in our experiments.

The management of endogenous control processes, based on internal representations, and exogenous control processes, based on stimulus repetitions (Experiment 1) and stimulus availability (Experiment 2), to guide random task choice occurred in a similar manner regardless of WMC. The implications of these findings are that task selection does not make large demands on the management of these types of information and processes, including the maintenance and updating of the sequence of tasks just performed or the need for resolution of competition between multiple sources of information, at least under the conditions used in these experiments. In fact, the results of Experiment 2 suggest that multiple sources of information can be processed and used to guide task selection in a noncompetitive way. Further, those with lower WMC can shift task selection between these various sources of information as easily as those with higher WMC.

This is not to say that task choice does not require endogenous control. Indeed, several pieces of evidence suggest that some endogenous control was required in our SOA experiment (Experiment 2). First, participants were instructed to make choices as if imagining a coin flip, an endogenous process. Second, on 0-ms SOA trials, individuals could not use $\mathrm{S} 1$ to guide task selection, but still abandoned the previously executed task and switched to a new one on almost $40 \%$ of trials, thus suggesting that endogenous choice processes were being used at least to replace the previously chosen task (Vandamme, Szmalec, Liefooghe, \& Vandierendonck in press). Third, even at the $150-\mathrm{ms}$ SOA, individuals only chose the S1 task on $60 \%$ of trials, only $10 \%$ above the probability of choosing S1 if individuals were not allowing the $\mathrm{S} 1$ to guide behavior. Therefore, on at least $40 \%$ of the trials individuals were basing task choice on something other than the identity of $\mathrm{S} 1$.

When multiple sources of information are available to guide different task choices, the choice may be determined 
by which of the possible sources of information is most active at some decision point. Each information source (previous trial task choice, random choice approximation, and availability of a current stimulus) loses or gains activation during the preparation interval without the need for additional WM resources (e.g., to resolve crosstalk interference; Gilbert \& Shallice, 2002). When a choice is required, the task indicated by the source with the most activation is chosen. In this view, task choice is not WM-dependent.

Switch probability was not correlated with WMC in Experiment 2, and in Experiment 1 the relationship of WMC to switch probability was only marginally significant. If WMC does influence task choice, it has a much smaller effect than would be expected based on the view that task choice processing uses multiple endogenous control mechanisms, such as the availability and representativeness heuristics and the inhibition of previous task goals. These findings suggest that it is necessary to reconsider the processes involved in task selection in the VTS and more generally in volitional control of behavior, in line with the non-WM-dependent view described above.

The results of these experiments stand in contrast to those of Arrington and Yates (2009), who found that the executive control measure of the ANT was correlated with switch probability $(r \mathrm{~s}=-.273$ and -.368 for the 200 - and 1,000-ms RSI conditions, respectively) but not with switch cost ( $r \mathrm{~s}=.016$ and .042 , respectively). In that study, executive control was measured as the difference between RTs on trials with congruent and incongruent flankers. It may be that the ANT measure of executive control assesses different cognitive mechanisms than the OSPAN. Heitz and Engle (2007) found that WMC as measured by the OSPAN is related to differences in the time available to focus spatial attention on the target stimulus in a flanker task. Attentional focusing was not a cognitive requirement in the VTS procedures used here, though. Further, Redick and Engle (2006) found that flanker and cuing manipulations in the ANT thought to independently affect executive control and spatial-orienting attentional networks, respectively, interacted, suggesting some shared processing components. Additionally, Redick and Engle pointed out that the measurement of attentional processing mechanisms through computation of difference scores leads to unreliable measurement. In contrast, task choice measures and the OSPAN task used in these experiments are highly reliable (see Tables 1 and 3).

\section{Conclusion}

The present findings add to a growing number of studies that have used the VTS procedure to show dissociations between task choice and task performance measures. These findings are consistent with models of cognitive control that represent task choice and task execution processes as different types of representations making different demands on executive control. One such model is the executive control theory of visual attention (ECTVA; Logan \& Gordon, 2001). Within the framework of ECTVA, choosing what task to do or goal to work toward would be equivalent to establishing a task-level representation, maintained as a proposition in WM. This process is different from the set of processes that establishes specific parameters, a parameter-level task set, that can lead to the completion of the task or realization of the goal. While the present results suggest that the process of forming a tasklevel representation is influenced by both external and internal factors, we found little evidence that the processes involved in making decisions to switch or repeat a task taxed WMC, at least not to the extent of seeing significant individual differences. In VTS and other multitask environments, task performance requires executive processing to reset control parameters following a response to prevent perseveration (i.e., lower switch probability) when the next response is made. At the level of setting specific task parameters, executive functioning operates to pass control parameters for the selected task from WM to the subordinate systems in TVA. Poor performance of this function by those with lower WMC might be the source of their slower and less accurate task performance. The emergence of a relationship between WMC and switch costs when short RSIs were mixed with longer RSIs suggests that this executive control function is temporally dependent (also see Logan \& Gordon, 2001): Those high in WMC were able to retrieve new task parameters (on switch trials) more effectively when they were needed shortly after the previous response, but those with reduced WMC were less effective at this.

These experiments suggest a minimal role of WMC in multitask environments with multiple sources of information that can guide adequate task performance. Those individuals that are lower in WMC are just as efficient at varying their task choices as those with more WMC. Further research is needed to examine the WM dependence of the various other strategies and mechanisms involved in selection of volitional behaviors in multitask environments. However, low WMC does impair the execution of those choices under time pressure, through delays and errors in the retrieval of the response rules when a new task is to be performed, and through poorer representations of the response rules that lead to more error-prone and slower responding on all trials. In sum, those low in WMC may be just as able to move between different tasks using their endogenous control abilities, but they are less efficient at executing those tasks. 
Acknowledgements Our thanks to Eric Ruthruff, Michael Kane, Klaus Oberauer, and anonymous reviewers for helpful feedback on this article. We also thank Melakeh Kurdi for her assistance with data collection and Bryan Franks for his help with the preparation of the manuscript.

\section{Appendix A}

Fig. 1 Scatterplots of RT switch costs by WMC in each Experiment, separated for RSIs
$100 \mathrm{~ms}$ RSI Experiment 1

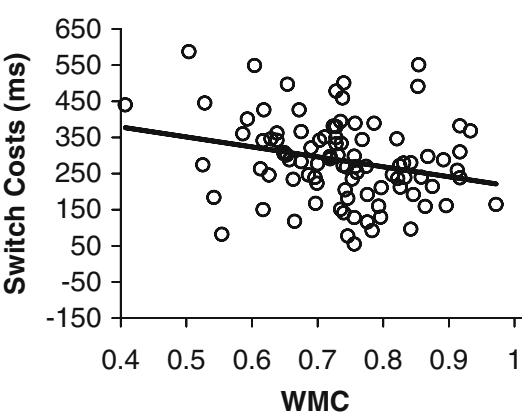

$900 \mathrm{~ms}$ RSI
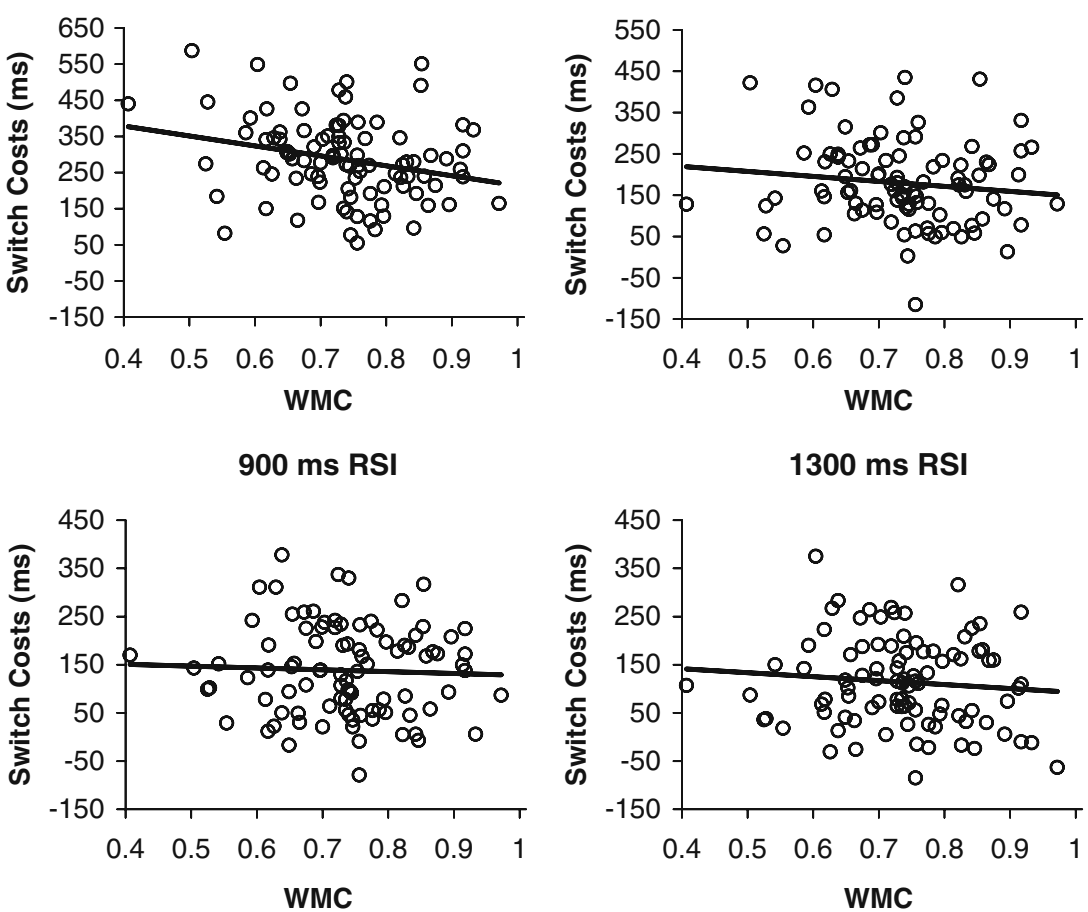

1300 ms RSI

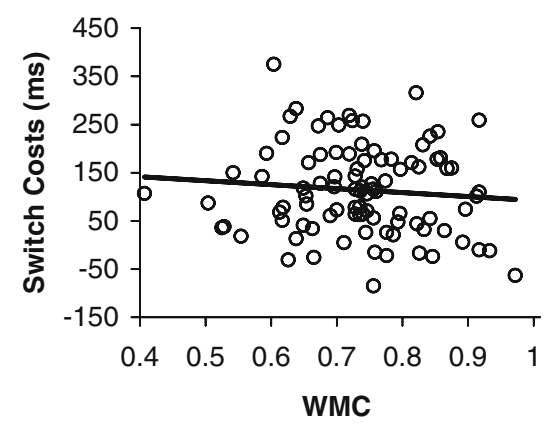

Experiment 2

500 ms RSI

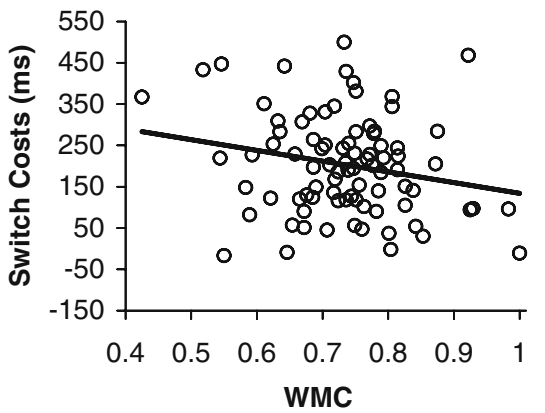

1300 ms RSI

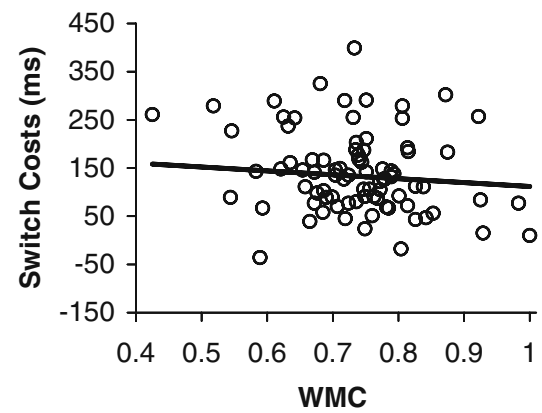


Fig. 2 Scatterplots of WMC by $p(\mathrm{~S} 1)$ in the $500-$ and $1,300-\mathrm{ms}$ RSI conditions of Experiment 2. Regression lines are plotted with $95 \%$ confidence intervals. The data point that was excluded as an outlier is marked with an $\mathrm{X}$

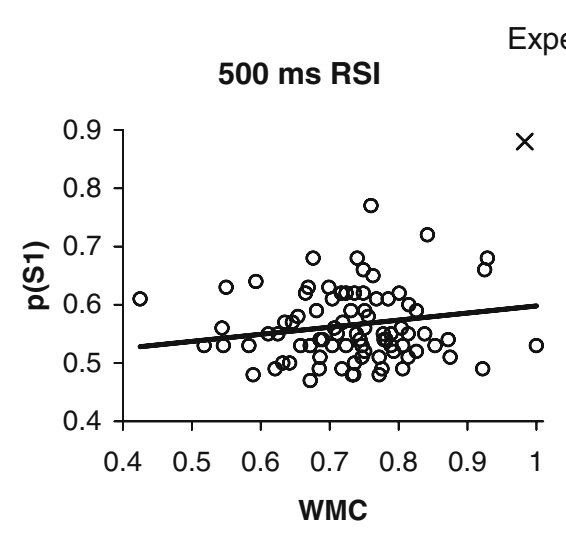

Experiment 2

1300 ms RSI

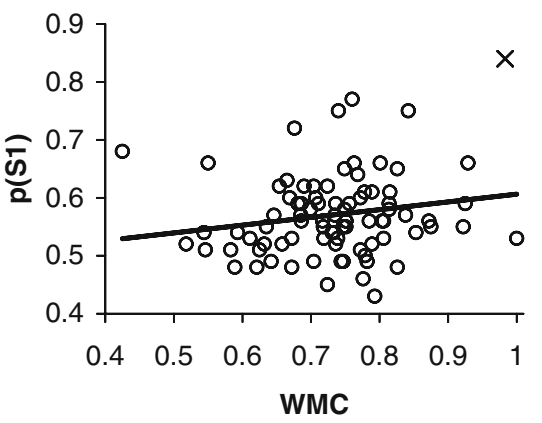

\section{References}

Allport, A., \& Wylie, G. (2000). Task switching, stimulus-response bindings, and negative priming. In S. Monsell \& J. Driver (Eds.), Control of cognitive processes: Attention and performance XVIII (pp. 35-70). Cambridge: MIT Press.

Altmann, E. M. (2004). The preparation effect in task switching: Carryover of SOA. Memory \& Cognition, 32, 153-163.

Altmann, E. M., \& Gray, W. D. (2008). An integrated model of cognitive control in task switching. Psychological Review, 115, 602-639. doi:10.1037/0033-295X.115.3.602

Arrington, C. M. (2008). The effect of stimulus availability on task choice in voluntary task switching. Memory \& Cognition, 36, 991-997. doi:10.3758/MC.36.5.991

Arrington, C. M., \& Logan, G. D. (2004). The cost of a voluntary task switch. Psychological Science, 15, 610-615. doi:10.1111/j.09567976.2004.00728.x

Arrington, C. M., \& Logan, G. D. (2005). Voluntary task switching: Chasing the elusive homunculus. Journal of Experimental Psychology. Learning, Memory, and Cognition, 31, 683-702. doi: $10.1037 / 0278-7393.31 .4 .683$

Arrington, C. M., Altmann, E. M., \& Carr, T. H. (2003). Tasks of a feather flock together: Similarity effects in task switching. Memory \& Cognition, 31, 781-789.

Arrington, C. M., Logan, G. D., \& Schneider, D. W. (2007). Separating cue encoding from target processing in the explicit task-cuing procedure: Are there "true" task switch effects? Journal of Experimental Psychology. Learning, Memory, and Cognition, 33, 484-502. doi:10.1037/0278-7393.33.3.484

Arrington, C. M., \& Yates, M. M. (2009). The role of attentional networks in voluntary task switching. Psychonomic Bulletin \& Review, 16, 660-665. doi:10.3758/PBR.16.4.660

Arrington, C. M., Weaver, S. M., \& Pauker, R. L. (2010). Stimulusbased priming of task choice during voluntary task switching. Journal of Experimental Psychology. Learning, Memory, and Cognition, 36, 1060-1067. doi:10.1037/a0019646

Baddeley, A., Chincotta, D., \& Adlam, A. (2001). Working memory and the control of action: Evidence from task switching. Journal of Experimental Psychology: General, 130, 641-657. doi:10. 1037/0096-3445.130.4.641

Baddeley, A., Emslie, H., Kolodny, J., \& Duncan, J. (1998). Random generation and the executive control of working memory. The Quarterly Journal of Experimental Psychology, 51A, 819-852. doi: $10.1080 / 027249898391413$

Brown, J. W., Reynolds, J. R., \& Braver, T. S. (2007). A computational model of fractionated conflict-control mechanisms in task-switching. Cognitive Psychology, 55, 37-85. doi:10.1016/ j.cogpsych.2006.09.005
Cohen, J. (1988). Statistical power analysis for the behavioral sciences (2nd ed.). Hillsdale: Erlbaum.

Conway, A. R. A., Cowan, N., \& Bunting, M. F. (2001). The cocktail party phenomenon revisited: The importance of working memory capacity. Psychonomic Bulletin \& Review, 8, 331-335.

Conway, A. R. A., Kane, M. J., Bunting, M. F., Hambrick, D. Z., Wilhelm, O., \& Engle, R. W. (2006). Working memory span tasks: A methodological review and user's guide. Psychonomic Bulletin \& Review, 12, 769-786.

De Jong, R. (2000). An intention-activation account of residual switch costs. In S. Monsell \& J. Driver (Eds.), Control of cognitive processes: Attention and performance XVIII (pp. 357-376). Cambridge: MIT Press.

Demanet, J., Verbruggen, F., Liefooghe, B., \& Vandierendonck, A. (2010). Voluntary task switching under load: Contribution of top-down and bottom-up factors in goal-directed behavior. Psychonomic Bulletin \& Review, 17, 387-393. doi:10.3758/ PBR.17.3.387

Fan, J., McCandliss, B. D., Sommer, T., Raz, A., \& Posner, M. I. (2002). Testing the efficiency and independence of attentional networks. Journal of Cognitive Neuroscience, 14, 340-347. doi:10.1162/089892902317361886

Gilbert, S. J., \& Shallice, T. (2002). Task switching: A PDP model. Cognitive Psychology, 44, 297. doi:10.1006/cogp.2001.0770

Heitz, R. P., \& Engle, R. W. (2007). Focusing the spotlight: Individual differences in visual attention control. Journal of Experimental Psychology: General, 136, 217-240. doi:10.1037/0096-3445. 136.2.217

Hester, R., \& Garavan, H. (2005). Working memory and executive function: The influence of content and load on the control of attention. Memory \& Cognition, 33, 221-233.

Kane, M. J., Bleckley, M. K., Conway, A. R. A., \& Engle, R. W. (2001). A controlled-attention view of working-memory capacity. Journal of Experimental Psychology: General, 130, 169-183. doi:10.1037/0096-3445.130.2.169

Kane, M. J., Conway, A. R. A., Hambrick, D. Z., \& Engle, R. W. (2007). Variation in working memory capacity as variation in executive attention and control. In A. R. A. Conway, C. Jarrold, M. J. Kane, A. Miyake, \& J. N. Towse (Eds.), Variation in working memory (pp. 21-46). New York: Oxford University Press.

Kane, M. J., \& Engle, R. W. (2003). Working-memory capacity and the control of attention: The contributions of goal neglect, response competition, and task set to Stroop interference. Journal of Experimental Psychology: General, 132, 47-70. doi:10.1037/ 0096-3445.132.1.47

Kane, M. J., Poole, B. J., Tuholski, S. W., \& Engle, R. W. (2006). Working memory capacity and the top-down control of visual search: Exploring the boundaries of "executive attention. Journal 
of Experimental Psychology. Learning, Memory, and Cognition, 32, 749-777. doi:10.1037/0278-7393.32.4.749

Koch, I., Gade, M., Schuch, S., \& Philipp, A. M. (2010). The role of inhibition in task switching: A review. Psychonomic Bulletin \& Review, 17, 1-14. doi:10.3758/PBR.17.1.1

Liefooghe, B., Barrouillet, P., Vandierendonck, A., \& Camos, V. (2008). Working memory costs of task switching. Journal of Experimental Psychology. Learning, Memory, and Cognition, 34, 478-494. doi:10.1037/0278-7393.34.3.478

Liefooghe, B., Demanet, J., \& Vandierendonck, A. (2009). Is advance reconfiguration in voluntary task switching affected by the design employed? The Quarterly Journal of Experimental Psychology, 62, 850-857. doi:10.1080/17470210802570994

Lien, M.-C., \& Ruthruff, E. (2008). Inhibition of task set: Converging evidence from task choice in the voluntary task-switching paradigm. Psychonomic Bulletin \& Review, 15, 1111-1116. doi:10.3758/PBR.15.6.1111

Logan, G. D. (1985). Skill and automaticity: Relations, implications, and future directions. Canadian Journal of Psychology, 39, 367386. doi:10.1037/h0080066

Logan, G. D. (2004). Working memory, task switching, and executive control in the task span procedure. Journal of Experimental Psychology: General, 133, 218-236. doi:10.1037/0096-3445. 133.2.218

Logan, G. D., \& Bundesen, C. (2003). Clever homunculus: Is there an endogenous act of control in the explicit task-cuing procedure? Journal of Experimental Psychology: Human Perception and Performance, 29, 575-599. doi:10.1037/0096-1523.29.3.575

Logan, G. D., \& Gordon, R. D. (2001). Executive control of visual attention in dual-task situations. Psychological Review, 108, 393 434. doi:10.1037/0033-295X.108.2.393

Mayr, U., \& Bell, T. (2006). On how to be unpredictable: Evidence from the voluntary task-switching paradigm. Psychological Science, 17, 774-780. doi:10.1111/j.1467-9280.2006.01781.x

Mayr, U., \& Keele, S. W. (2000). Changing internal constraints on action: The role of backward inhibition. Journal of Experimental Psychology: General, 129, 4-26. doi:10.1037/0096-3445. 129.1.4

Mayr, U., \& Kliegl, R. (2000). Task-set switching and long-term memory retrieval. Journal of Experimental Psychology. Learning, Memory, and Cognition, 26, 1124-1140. doi:10.1037/02787393.26.5.1124

Mayr, U., \& Kliegl, R. (2003). Differential effects of cue changes and task changes on task-set selection costs. Journal of Experimental Psychology. Learning, Memory, and Cognition, 29, 362-372. doi:10.1037/0278-7393.29.3.362

Meiran, N. (1996). Reconfiguration of processing mode prior to task performance. Journal of Experimental Psychology. Learning, Memory, and Cognition, 22, 1423-1442. doi:10.1037/0278-7393. 22.6.1423

Miyake, A., Friedman, N. P., Emerson, M. J., Witzki, A. H., Howerter, A., \& Wager, T. D. (2000). The unity and diversity of executive functions and their contributions to complex "frontal lobe" tasks: A latent variable analysis. Cognitive Psychology, 41, 49-100. doi:10.1006/cogp.1999.0734

Monsell, S. (2003). Task switching. Trends in Cognitive Sciences, 7, 134-140. doi:10.1016/S1364-6613(03)00028-7
Oberauer, K., Süß, H.-M., Schulze, R., Wilhelm, O., \& Wittmann, W. W. (2000). Working memory capacity-Facets of a cognitive ability construct. Personality and Individual Differences, 29, 1017-1045. doi:10.1016/S0191-8869(99)00251-2

Oberauer, K., Süß, H.-M., Wilhelm, O., \& Wittman, W. W. (2003). The multiple faces of working memory: Storage, processing, supervision, and coordination. Intelligence, 31, 167-193. doi:10.1016/S0160-2896(02)00115-0

Rapoport, A., \& Budescu, D. V. (1997). Randomization in individual choice behavior. Psychological Review, 104, 603-617. doi:10.1037/0033-295X.104.3.603

Redick, T. S., \& Engle, R. W. (2006). Working memory capacity and attention network test performance. Applied Cognitive Psychology, 20, 713-721. doi:10.1002/acp.1224

Rogers, R. D., \& Monsell, S. (1995). Costs of a predictable switch between simple cognitive tasks. Journal of Experimental Psychology: General, 124, 207-231. doi:10.1037/0096-3445. 124.2.207

Rosen, V. M., \& Engle, R. W. (1998). Working memory capacity and suppression. Journal of Memory and Language, 39, 418. doi:10.1006/jmla.1998.2590

Schneider, D. W., \& Logan, G. D. (2005). Modeling task switching without switching tasks: A short-term priming account of explicitly cued performance. Journal of Experimental Psychology: General, 134, 343-367. doi:10.1037/0096-3445.134.3.343

Schneider, W., Eschmann, A., \& Zuccolotto, A. (2002). E-Prime user's guide. Pittsburgh: Psychology Software Tools, Inc.

Sobel, K. V., Gerrie, M. P., Poole, B. J., \& Kane, M. J. (2007). Individual differences in working memory capacity and visual search: The roles of top-down and bottom-up processing. Psychonomic Bulletin \& Review, 14, 840-845.

Towse, J. N., \& Neil, D. (1998). Analyzing human random generation behavior: A review of methods used and a computer program for describing performance. Behavior Research Methods, Instruments, \& Computers, 30, 583-591.

Turner, M. L., \& Engle, R. W. (1989). Is working memory capacity task dependent? Journal of Memory and Language, 28, 127-154. doi:10.1016/0749-596X(89)90040-5

Unsworth, N., \& Engle, R. W. (2007). The nature of individual differences in working memory capacity: Active maintenance in primary memory and controlled search from secondary memory. Psychological Review, 114, 104-132. doi:10.1037/0033-295X. 114.1.104

Vandamme, K., Szmalec, A., Liefooghe, B., \& Vandierendonck, A. (in press). Are voluntary switches corrected repetitions? Psychophysiology.

Vandierendonck, A., Liefooghe, B., \& Verbruggen, F. (2010). Task switching: Interplay of reconfiguration and interference control. Psychological Bulletin, 136, 601-626. doi:10.1037/a0019791

Weaver, S. M., \& Arrington, C. M. (2010). What's on your mind: The influence of the contents of working memory on choice. The Quarterly Journal of Experimental Psychology, 63, 726-737. doi:10.1080/17470210903137180

Yeung, N. (2010). Bottom-up influences on voluntary task switching: The elusive homunculus escapes. Journal of Experimental Psychology. Learning, Memory, and Cognition, 36, 348-362. doi:10.1037/a0017894 